\title{
On a Class of Parametric Transforms and Its Application to Image Compression
}

\author{
Susanna Minasyan, ${ }^{1}$ Jaakko Astola, ${ }^{1}$ and David Guevorkian ${ }^{2}$ \\ ${ }^{1}$ Institute of Signal Processing, Tampere University of Technology (TUT), P.O. Box 527, 33101 Tampere, Finland \\ ${ }^{2}$ Nokia Research Center, P.O. Box 100, 33721 Tampere, Finland
}

Received 14 July 2006; Revised 29 January 2007; Accepted 27 April 2007

Recommended by Mauro Barni

\begin{abstract}
A class of parametric transforms that are based on unified representation of transform matrices in the form of sparse matrix products is described. Different families of transforms are defined within the introduced class. All transforms of one family can be computed with fast algorithms similar in structure to each other. In particular, the family of Haar-like transforms consists of discrete orthogonal transforms of arbitrary order such that they all may be computed with a fast algorithm that is in structure similar to classical fast Haar transform. A method for parameter selection is proposed that allows synthesizing specific transforms with matrices containing predefined row $(\mathrm{s})$. The potential of the proposed class of Haar-like parametric transforms to improve the performance of fixed block transforms in image compression is investigated. With this purpose, two image compression schemes are proposed where a number of Haar-like transforms are synthesized each adapted to a certain set of blocks within an image.The nature of the proposed schemes is such that their performance (in terms of PSNR versus compression ratio) cannot be worse than a scheme based on classical discrete cosine transform (DCT). Simulations show that a significant performance improvement can be achieved for certain types of images such as medical X-ray images and compound images.
\end{abstract}

Copyright (C) 2007 Susanna Minasyan et al. This is an open access article distributed under the Creative Commons Attribution License, which permits unrestricted use, distribution, and reproduction in any medium, provided the original work is properly cited.

\section{INTRODUCTION}

Many traditional image compression methods are based on fixed transforms. Early image compression systems were based on splitting images into fixed-size blocks and applying a fixed block transform to each block. For example, the JPEG image compression standard [1] is based on the discrete cosine transform (DCT). Also other fixed block transforms like Fourier, Walsh, Haar, and Sine transforms, were used for special types of images [2-13]. Modern image compression systems support the JPEG2000 standard [14], which is based on wavelet transforms that are applied to whole image.

The wavelet based image compression methods are commonly known to outperform the block transform-based methods [15]. However, the block transform-based methods still have potential of being improved by making them more adaptive to image content. For example, in [16], a DCTbased image compression method is proposed that significantly outperforms the JPEG2000, in particular, by adapting the block size (and the DCT size) according to activity of the image content in different regions. Let us, however, note that the used transform is fixed and only its size is adapted to image in the method of [16].

A potential of further improving the performance of image compression methods by adapting the used transforms to different image regions may exist since each fixed transform is optimal for a specific class of inputs but none of them may provide satisfactory solution to a wide range of possible inputs. From this point of view, parametric transforms with matrices described in a unified form and based on a set of parameters have become important. In this context, parametric transform, actually, means a wide class of discrete orthogonal transforms (DOTs) that may include classical transforms and an infinite number of new transforms with the possibility to select the desired transform according to parameter values. A unified software/hardware tool can be used to implement the whole class of transforms with the possibility to adapt a transform by varying the parameters. Various methods to synthesize parametric transforms have been developed in $[2,4-6,11-13,17-23]$.

In [20-23], a class of parametric transforms, matrices of which have a unified representation in the form of products 
of sparse block-diagonal matrices and permutation matrices, has been proposed. Several families of transforms have been defined within this class such that all transforms of one family can be computed with a fast algorithm of the same structure. In particular, a family of Haar-like transforms that can all be computed with fast algorithm in structure similar to classical fast Haar transform algorithm has been proposed. A methodology to synthesize a Haar-like transform such that its matrix contains desired predefined basis function(s) has also been developed (first presented in [22] and later also in [23]). Similarly, a family of Hadamard-like transforms and a family of slant-like transforms have been introduced in $[22,23]$.

The general goal of this paper is to analyze the potential advantages of adaptive transform-based image compression methods over the fixed transform-based ones. Specifically, a class of parametric transforms is defined and its potential to improve block transform-based image compression methods is investigated. With this aim, two parametric Haar-like transform-based adaptive image compression algorithms are proposed and their performances are compared to the performance of the similar algorithm that is based on the fixed DCT. In both algorithms, the classical DCT is used along with new transforms that are synthesized according to input image within the defined parametric class of transforms. The first algorithm is based on an iterative scheme where the classical DCT is used at the first iteration and iteratively synthesized Haar-like transforms are used at the following iterations to refine the compression quality. The process is iterated as long as a rewarding performance improvement may be achieved. In the second compression algorithm the classical DCT transform and several image dependent Haar-like transforms are applied in parallel to each nonoverlapping $8 \times 8$ block of the input image. The transform that achieves the best result is then selected to be assigned to the corresponding block. Let us note that both compression schemes have a performance that at least cannot be worse than a DCTbased compression scheme. Extensive simulations were conducted to compare the performance of the parametric Haarlike transform-based adaptive image compression methods with the performance of the similar algorithm that is based on fixed DCT. Several types of images were simulated. Experiments illustrated a moderate performance improvement for natural images and significant performance improvement for images of certain types such as medical images and complex images consisting of fragments of essentially different types.

The paper is organized as follows. In Section 2 we review the parametric representation of a general class of fast transforms and define families of Haar-like, Hadamard-like, and slant-like transforms. A methodology of synthesizing parametric transforms of arbitrary order with one or more predefined basis function(s) is also presented in this section. The proposed image compression algorithms are described in Section 3. The results of experiments and performance analysis of the algorithms are given in Section 4. A unified hardware architecture to synthesize and implement the fast parametric transforms is presented in Section 5. The conclusions are given in Section 6.

\section{GENERALIZED FAST PARAMETRIC TRANSFORMS}

In this section we present a unified parametric representation of widely used fast algorithms for many fixed discrete orthogonal transforms in a generalized form for arbitrary order (size of the transform matrix). This approach not only allows of describing many existing fast transform algorithms in a unified form but also gives an opportunity to synthesize a broad family of new orthogonal transforms a priori having fast algorithms for their computation. In particular, families of Haar-like, Hadamard-like, and slant-like transforms are defined based on this approach.

\subsection{The class of parametric transforms}

Let $H_{N}$ be an orthogonal $(N \times N)$-matrix and let

$$
\mathbf{y}=H_{N} \cdot \mathbf{x}
$$

be the corresponding discrete orthogonal transform (DOT) of $(N \times 1)$-vector $\mathbf{x}$.

The inverse DOT is defined as

$$
\mathbf{x}=\frac{1}{N} H_{N}^{*} \cdot \mathbf{y},
$$

where $H_{N}^{*}$ is the conjugate transpose of the matrix $H_{N}$. Obviously, the computational complexities of both the direct DOT (1) and the inverse DOT (2) are estimated as $C($ DOT $)=O\left(N^{2}\right)$ operations in the general case. In practical applications, faster real-time computation is needed. Therefore, numerous fast algorithms have been developed for different fixed DOTs, for example, the well-known fast fourier transforms (FFT), fast cosine transforms (FDCT), fast Walsh-Hadamard transform (FWHT), fast Haar transform (FHT), and so forth (see, e.g., [2-8]). Analyzing these algorithms one can see that most of them can be described in a unified form. In [2, 4-6, 11-13, 17-21] several unified representations of the fast transform algorithms are described. These representations can be generalized (see $[20,21]$ ) to the following representation of the transform matrix as the product:

$$
H_{N}=P^{(m+1)} \prod_{j=m}^{1} H^{(j)} P^{(j)}
$$

of sparse matrices $H^{(j)}, j=1, \ldots, m$, which are $(N \times N)$ block-diagonal matrices with square blocks (spectral kernels) on the main diagonal, and $P^{(j)}, j=1, \ldots, m+1$, are $(N \times N)$ permutation matrices. ${ }^{1}$

Typically, the order $N$ of the fast transform is considered to be a composite number (most often a power of two or of another integer). Even though the composite number

\footnotetext{
${ }^{1}$ A permutation matrix is a matrix obtained by permuting the rows of an $n \times n$ identity matrix according to some permutation of the numbers 1 to $n$. Every row and column therefore contain precisely a single 1 with 0 's everywhere else.
} 


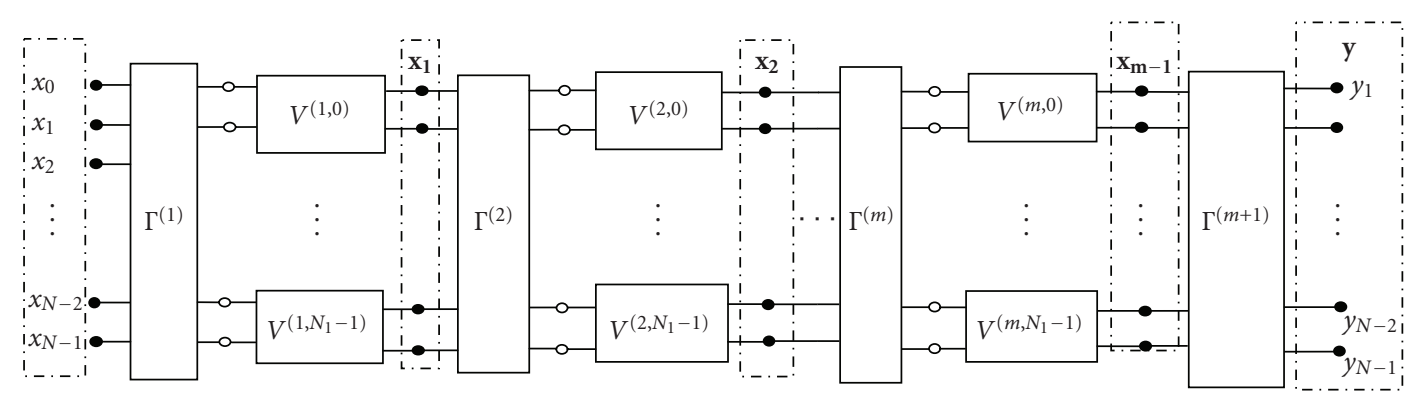

(a)

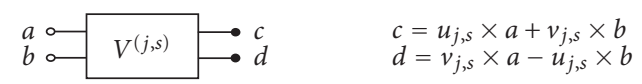

$$
V^{(j, s)}=\left[\begin{array}{cc}
u_{j, s} & v_{j, s} \\
v_{j, s} & -u_{j, s}
\end{array}\right]
$$

(b)

FIgURE 1: The unified flowgraph of fast transform algorithms: (a) flowgraph structure; (b) the basic (butterfly) operation.

can formally be an arbitrary positive integer, the efficiency of the corresponding fast algorithm is not high for numbers presented with a small number of prime factors. In particular, if $N$ is a prime number, the corresponding "fast" algorithm becomes, in fact, the direct matrix-vector multiplication method with the computational complexity of $O\left(N^{2}\right)$.

Here we define a class of parametric transforms of arbitrary order $N$ such that the efficiency of the corresponding fast algorithm does not depend on the number of factors of $N$.

Definition 1. Define the class $\Omega$ of discrete orthogonal transforms such that their matrices may be presented in the form of (3), where $P^{(j)}, j=1, \ldots, m+1$, are $(N \times N)$ permutation matrices, and $H^{(j)}, j=1, \ldots, m$, are $(N \times N)$ block-diagonal matrices of the form

$$
H^{(j)}=\left(\bigoplus_{s=0}^{k} V^{(j, s)}\right) \oplus I_{N \bmod 2} \oplus\left(\bigoplus_{s=k+1}^{\lceil N / 2\rceil} V^{j, s}\right),
$$

where $k \in\{0,1, \ldots,\lceil N / 2\rceil-1\}, V^{(j, s)}$ are $(2 \times 2)$ matrices called spectral kernels, $I_{p}$ is either an identity matrix of order 1 if $p=1$ or an empty matrix if $p=0$, the sign $\oplus$ stands for the direct sum of matrices, and the sign $\lceil a\rceil$ for the smallest integer larger or equal to $a$.

Obviously, the transform matrix presented in the form (3) is orthogonal if the spectral kernels in (4) are orthogonal. It should be noted that the spectral kernels and the permutation matrices as well as the numbers $m$ and $k$ play the role of parameters varying which different transforms from $\Omega$ may be synthesized. In other words, by choosing various sets of permutation matrices and spectral kernels, it is possible to synthesize various orthogonal bases $H_{N}$ produced from (3).

Transforms from $\Omega$ can be computed with a fast algorithm in $m$ iterative stages:

$$
\begin{gathered}
\mathbf{x}_{0}=\mathbf{x} ; \quad \mathbf{x}_{j}=H^{(j)} \cdot\left(P^{(j)} \mathbf{x}_{j-1}\right), \quad j=1, \ldots, m ; \\
\mathbf{y}=P^{(m+1)} \mathbf{x}_{m} .
\end{gathered}
$$

At the stage $j=1, \ldots, m$; the input vector $\mathbf{x}_{j-1}$ to that stage is first permuted according to $P^{(j)}$ and then the result is multiplied by the block diagonal matrix $H^{(j)}$, which is equivalent to multiplying the corresponding $(2 \times 2)$ spectral kernels to corresponding $(2 \times 1)$ subvectors of the permuted vector. Since the matrix $H^{(j)}, j=1, \ldots, m$, contains at most $4\lceil N / 2\rceil \approx 2 N$ nonzero entries, the complexity of the algorithm (5) is estimated as $O(m N)$ operations at the most instead of $O\left(N^{2}\right)$ in the direct method. Thus, the transforms from $\Omega$ possess fast algorithms.

The fast transform algorithm (5) may nicely be presented by the flowgraph, generically illustrated in Figure 1. The nodes of the flowgraph (bold dotes) are divided into $m+1$ levels, the $j$ th level representing the vector $\mathbf{x}_{j}$, $j=0, \ldots, m$, from (5). There are directed edges only between nodes of adjacent levels. Sets of edges denoted $\Gamma^{(1)}, \Gamma^{(2)}, \ldots, \Gamma^{(m+1)}$ in Figure 1 correspond to permutation matrices $P^{(1)}, P^{(2)}, \ldots, P^{(m+1)}$ so that the outputs of the $\Gamma^{(j)}$ block (which are marked with small circles), represent the vector $\mathbf{x}_{j}=\left(P^{(j)} x_{j-1}\right)$. Blocks $V^{(j, s)}, j=1, \ldots, m, s=$ $0, \ldots, N_{j}-1$, on Figure 1, represent executions of the basic operations, which are simple 2-point discrete orthogonal transforms (multiplication of a $2 \times 2$ orthogonal matrix by a 2 -point vector or "butterfly"). Recall that the general form of an orthogonal $2 \times 2$ matrix is

$$
V=\left[\begin{array}{cc}
u & v \\
v & -u
\end{array}\right]
$$

where $u^{2}+v^{2}=1$ and the "minus" sign may float to any of the four entries.

Let us now consider two families of transforms within $\Omega$, the families of Hadamard-like and Haar-like transforms, which are of a particular interest since they are generalizations of the classical Hadamard and Haar transforms.

Definition 2. Within the class $\Omega$ consider the family $\bar{\Omega}$ of Hadamard-like orthogonal transforms such that all the spectral kernels are orthogonal with all nonzero entries. 


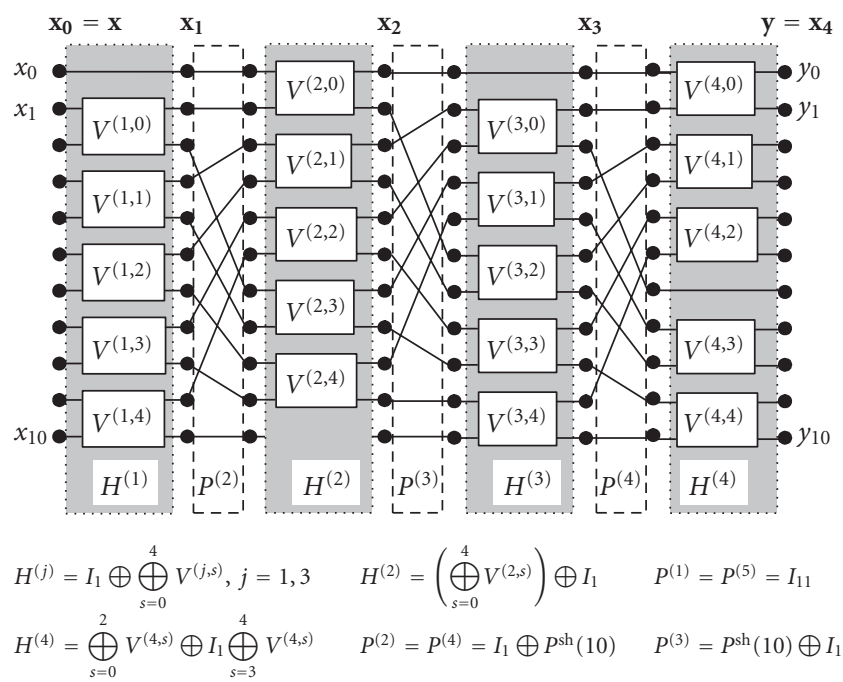

FIGURE 2: The fast Hadamard-like transform of order $N=11$.

The classical Hadamard transform belongs to the family $\bar{\Omega}$ of Hadamard-like transforms and corresponds to the following choice of the parameters: $m=\log _{2} N, N=2^{m}, k \equiv 0$, $P^{(j)}, j=1, \ldots, m$, are all the perfect shuffle permutation matrices, ${ }^{2}$ and

$$
V^{(j, s)}=\frac{1}{\sqrt{2}} \cdot\left[\begin{array}{cc}
1 & 1 \\
1 & -1
\end{array}\right],
$$

$j=1, \ldots, m, s=0, \ldots, N / 2-1$. Let us note that multiplications with the coefficient $1 / \sqrt{2}$ may be collected from stage to stage to be pre- or post-performed before or after the fast Hadamard transform algorithm. Therefore, only additions/subtractions will be performed during this algorithm.

An example of a new Hadamard-like fast transform flowgraph of order $N=11$ is shown in Figure 2 .

Note that for transforms from $\bar{\Omega}$, every matrix $H^{(j)}$, $j=1, \ldots, m$, contains exactly $4\lceil N / 2\rceil \approx 2 N$ nonzero entries. Therefore, the complexity of the algorithm (5) is estimated as $O(m N)$ for transforms from $\bar{\Omega}$.

Definition 3. Within the class $\Omega$ consider the family $\underline{\Omega}$ of Haar-like orthogonal transforms such that all the spectral kernels are orthogonal and

(1) all the entries of the spectral kernels $V^{(j, s)}, j=$ $1, \ldots, m$, are nonzero for $s=0, \ldots, N_{j}-1$, where $N_{j}=\overbrace{\lceil\cdots\lceil N / 2\rceil / 2 / \cdots / 2\rceil}^{j \text { times }}$;

(2) $V^{(j, s)}=I_{2}$ for $s=N_{j}, \ldots,\lceil N / 2\rceil-1, j=1, \ldots, m$;

(3) $P^{(j)}=P_{1}^{(j)} \oplus I_{N-N_{j}}$, where $P_{1}^{(j)}$ is a permutation matrix of order $N_{j}$.

\footnotetext{
2 The perfect shuffle operator of order $2 N$ collects all $N$ even components onto the first half and the $N$ odd components onto the second half of the output vector.
}

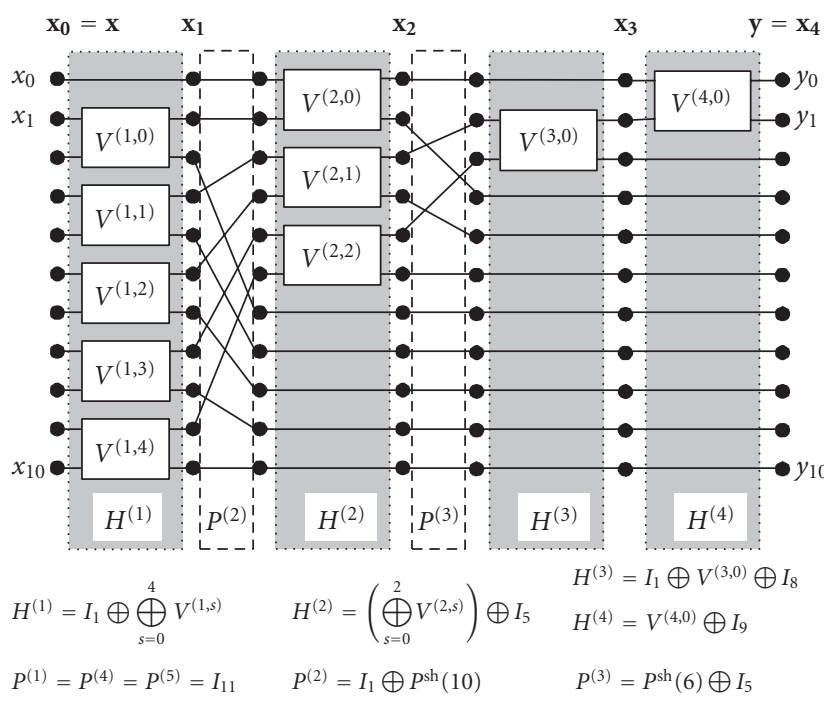

Figure 3: The fast Haar-like transform of order $N=11$.

Haar transform is the classical representative of $\underline{\Omega}$. It is obtained by taking $m=\log _{2} N, k \equiv 0, P_{1}^{(j)}, j=1, \ldots, m$, is the perfect shuffle permutation matrix of order $N_{j}=\left\lceil N / 2^{j}\right\rceil$, and $V^{(j, s)}=(1 / \sqrt{2})\left[\begin{array}{cc}1 & 1 \\ 1 & -1\end{array}\right], j=1, \ldots, m, s=0, \ldots, N / 2^{j}-1$. Again, multiplications to the coefficient $1 / \sqrt{2}$ may be collected from stage to stage to be pre- or post-performed.

An example of a Haar-like fast transform flowgraph of order $N=11$ is shown in Figure 3 .

Note that for transforms from $\underline{\Omega}$, the matrix $H^{(j)}, j=$ $1, \ldots, m$, contains only $4 N_{j} \approx N / 2^{j-3}$ nonzero entries. Therefore, the complexity of the algorithm of (5) is estimated as $O(N)$. Thus, the transforms from $\underline{\Omega}$ possess fast algorithms, which are even faster than those for the family $\bar{\Omega}$, for which the complexity is $O(m N)$. This can also be noted from the structures of the flowgraphs. While the flowgraphs of Hadamard-like transforms all have a "semirectangular" structure (equal number of nodes (butterflies) at every stage), the flowgraphs of Haar-like transforms all have "semitriangular" structure (approximately twice reduced number of nodes from a stage to the next).

\subsection{Synthesis of fast Haar-like and Hadamard-like transforms with a predefined basis functions}

We now present an algorithm for synthesizing a transform from $\Omega$, the matrix $H_{N}$ of which has an arbitrary predefined normalized vector $\mathbf{h}(\|\mathbf{h}\|=1)$ on its first row. Since $H_{N}$ should be orthogonal the latter condition means

$$
H_{N} \mathbf{h}^{T}=[1,0, \ldots, 0]^{T} .
$$

Therefore, the problem is reduced to defining the sets of edges and spectral kernels in a flowgraph of the structure shown on Figure 1 so that the first output $y_{0}$ of the flowgraph is unity and all the others are zero provided that the vector $\mathbf{h}$ was the input to the flowgraph. This may be achieved by the following algorithm, which iteratively approximately halves 
the number of nonzero input components at each of the $m$ stages until only one nonzero component is left.

Algorithm 1 (synthesis of $H_{N} \in \underline{\Omega}$ with $\mathbf{h}$ in the first row).

Step 1. Assume that the desired normalized vector $\mathbf{h}$ is the input to a flowgraph of a fast transform that has at least $\left\lceil\log _{2} N\right\rceil$ stages, the $j$ th stage, $j=1, \ldots, m$, consisting of at least $N_{j}=\left\lceil N / 2^{j}\right\rceil$ butterflies.

Step 2. For the first stage do the following.

Step 2.1. Arbitrarily define the set of edges $\Gamma^{(1)}$ (or equivalently, arbitrarily define the permutation matrix $\left.P^{(1)}\right)$.

Step 2.2. Define spectral kernels

$$
V^{(1, s)}=\frac{1}{\sqrt{\left(u_{1, s}\right)^{2}+\left(v_{1, s}\right)^{2}}}\left[\begin{array}{cc}
u_{1, s} & v_{1, s} \\
v_{1, s} & -u_{1, s}
\end{array}\right], \quad s=0, \ldots, N_{1}-1
$$

by assigning to the pair $\left[u_{1, s}, v_{1, s}\right]$ the values of the corresponding two components of the vector $\mathbf{h}$ that are input to the sth operation of the first stage of the flowgraph. If, however, both of these components are equal to zero, then arbitrarily define the corresponding spectral kernel. If only one of these components is nonzero, then define the corresponding spectral kernel to be an identity matrix of size $2 \times 2$. Note that this definition of spectral kernels implies that the second outputs of all butterflies are always zeros.

Step 2.3. Apply the first stage of the flowgraph to the input vector $\mathbf{h}$ and obtain vector $\mathbf{x}_{1}$.

Step 3. For every stage $j=2, \ldots, m$ do the following.

Step 3.1. Define the set of edges $\Gamma^{(j)}$ so that it passes the first (nonzero) outputs of butterflies of the previous stage to the inputs of uppermost butterflies of the current stage. Note that all the $\left\lceil N / 2^{j}\right\rceil$ nonzero outputs of the previous stage will be distributed among $N_{j}=\left\lceil N / 2^{j}\right\rceil$ butterflies of the previous stage.

Step 3.2. Define the spectral kernels

$$
V^{(j, s)}=\frac{1}{\sqrt{\left(u_{j, s}\right)^{2}+\left(v_{j, s}\right)^{2}}}\left[\begin{array}{cc}
u_{j, s} & v_{j, s} \\
v_{1, s} & -u_{j, s}
\end{array}\right],
$$

where $\left[u_{j, s}, v_{j, s}\right]$ are the corresponding two components of the vector $\mathbf{x}_{j-1}$ that are passed to the sth operation of the current stage of the flowgraph. Again if both of these components are equal to zero, then arbitrarily define the corresponding orthogonal kernel, and if only one of these components is nonzero, then define the corresponding spectral kernel to be an identity $2 \times 2$ matrix.

Step 3.3. Apply the $j$ th stage of the flowgraph to the vector $\mathbf{x}_{j-1}$, and obtain vector $\mathbf{x}_{j}$.

Step 4. Arbitrarily define the set of edges $\Gamma^{m+1}$ but so that it does not change the position of the first component.
Since the number of nonzero components approximately halves from stage to stage and since the number of stages is $m \geq \log _{2} N$, only the first output of the flowgraph will be nonzero and equal to unity (the input $\mathbf{h}$ was normalized). Thus, the corresponding transform: (a) will have an orthogonal matrix (spectral kernels were selected orthogonal); (b) may be computed with a fast algorithm; (c) will contain the desired predefined vector $\mathbf{h}$ in its first row.

Let us consider an example of synthesizing a Haar-like transform of order $N=8$ with the generating vector $\mathbf{h}=$ $(1 / \sqrt{204}) \cdot[1,2,3,4,5,6,7,8]$ as its first row. The matrix $H_{8}$ of the desired transform can be presented as

$$
H_{8}=P^{(4)} H^{(3)} P^{(3)} H^{(2)} P^{(2)} H^{(1)} P^{(1)},
$$

where we define $P^{(1)}=P^{(4)}=I_{8}$. Then, according to Step 2.2 of Algorithm 1 we define

$$
\begin{aligned}
H^{(1)}= & \frac{1}{\sqrt{5}}\left[\begin{array}{cc}
1 & 2 \\
2 & -1
\end{array}\right] \oplus \frac{1}{5}\left[\begin{array}{cc}
3 & 4 \\
4 & -3
\end{array}\right] \\
& \oplus \frac{1}{\sqrt{61}}\left[\begin{array}{cc}
5 & 6 \\
6 & -5
\end{array}\right] \oplus \frac{1}{\sqrt{113}}\left[\begin{array}{cc}
7 & 8 \\
8 & -7
\end{array}\right] .
\end{aligned}
$$

With this matrix we obtain the result of the first stage:

$$
\mathbf{x}_{\mathbf{1}}=H^{(1)} \mathbf{h}=\frac{1}{\sqrt{204}}[\sqrt{5}, 0,5,0, \sqrt{61}, 0, \sqrt{113}, 0]^{T} .
$$

We then define the permutation matrix $P^{(2)}=P^{\text {sh }}(8)$ to be the perfect shuffle of order 8 . Applying $P^{(2)}$ to $\mathbf{x}_{1}$ results in

$$
P^{(2)} \mathbf{x}_{1}=\left(\frac{1}{\sqrt{204}}\right) \cdot[\sqrt{5}, 5, \sqrt{61}, \sqrt{113}, 0,0,0,0]^{T} .
$$

Now, according to Step 3.2, we define $H^{(2)}$ as

$$
H^{(2)}=\frac{1}{\sqrt{30}}\left[\begin{array}{cc}
\sqrt{5} & 5 \\
5 & -\sqrt{5}
\end{array}\right] \oplus \frac{1}{\sqrt{174}}\left[\begin{array}{cc}
\sqrt{61} & \sqrt{113} \\
\sqrt{113} & -\sqrt{61}
\end{array}\right] \oplus I_{4} .
$$

Applying this matrix to $P^{(2)} \mathbf{x}_{1}$ yields

$$
\mathbf{x}_{\mathbf{2}}=H^{(2)} P^{(2)} \mathbf{x}_{\mathbf{1}}=\frac{1}{\sqrt{204}} \cdot[\sqrt{30}, 0, \sqrt{174}, 0,0,0,0]^{T}
$$

Taking $P^{(3)}=P^{(\mathrm{sh})}(4) \oplus I_{4}$ and defining

$$
H^{(3)}=\frac{1}{\sqrt{204}}\left[\begin{array}{cc}
\sqrt{30} & \sqrt{174} \\
\sqrt{174} & -\sqrt{30}
\end{array}\right] \oplus I_{6}
$$

we will find

$$
\mathbf{x}_{\mathbf{3}}=H^{(3)} P^{(3)} \mathbf{x}_{\mathbf{2}}=[1,0,0,0,0,0,0,0]^{T} .
$$




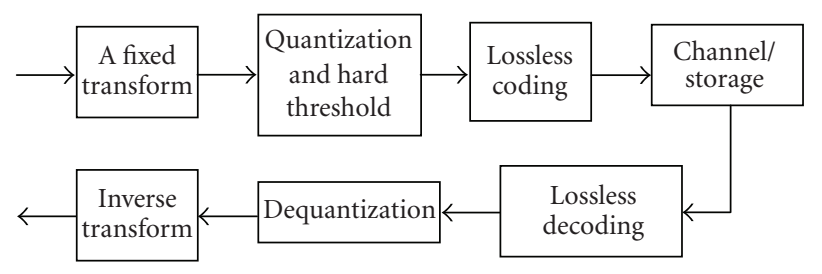

FIGURE 4: Generic transform-based image compression/decompression system.

Substituting the defined matrices into the factorization (11) of $H_{8}$ we obtain the desired transform matrix

$H_{8} \approx$

$\frac{1}{\sqrt{204}} \cdot\left[\begin{array}{cccccccc}1 & 2 & 3 & 4 & 5 & 6 & 7 & 8 \\ 2.4 & 4.8 & 7.2 & 9.6 & -2.1 & -2.5 & -2.9 & -3.3 \\ 5.8 & 11.7 & -3.5 & -4.7 & 0 & 0 & 0 & 0 \\ 0 & 0 & 0 & 0 & 7.4 & 8.8 & -5.6 & -6.4 \\ 12.8 & -6.4 & 0 & 0 & 0 & 0 & 0 & 0 \\ 0 & 0 & 11.4 & -8.6 & 0 & 0 & 0 & 0 \\ 0 & 0 & 0 & 0 & 10.9 & -9.1 & 0 & 0 \\ 0 & 0 & 0 & 0 & 0 & 0 & 10.7 & -9.4\end{array}\right]$.

Note that the obtained matrix has a structure similar to that of classical Haar transform matrix in the sense of distribution of positive, negative, and zero entries. At the same time it has the desired generating vector as its first row.

\subsection{Fast Haar-slant and Hadamard-slant transforms}

In this section, we further develop Algorithm 1 and construct orthogonal $N \times N$ matrices (arbitrary $N$ ) having the representations (3), (4) and involving more than one given orthogonal vectors as rows. In particular, one can consider the case of slant-like transforms wherein the first row of the matrix is the normalized constant vector $\mathbf{e}=$ $(1 / \sqrt{N}) \cdot[1,1, \ldots, 1]$ of length $N$, and the second row is a normalized slant (monotonically decreasing) vector $\mathbf{a}=$ $\left[\alpha_{0}, \alpha_{1}, \ldots, \alpha_{N-1}\right]$ of arbitrary slanting angle $\gamma=\tan ^{-1}\left(\alpha_{i+1}-\right.$ $\left.\alpha_{i}\right), i=0, \ldots, N-2$ and orthogonal to the first row. The matrix $H_{N}$ of the desired Haar-slant (or Hadamard-slant) transform may be found as the product

$$
H_{N}=\left(I_{1} \oplus H_{N-1}^{\prime \prime}\right) H_{N}^{\prime},
$$

where $H_{N}^{\prime} \in \underline{\Omega}$ (or $H_{N}^{\prime} \in \bar{\Omega}$ ) is a matrix having e at its first row, and $H_{N-1}^{\prime \prime} \in \underline{\Omega}\left(\right.$ or $\left.H_{N-1}^{\prime \prime} \in \bar{\Omega}\right)$ is an $(N-1) \times(N-1)$ matrix having the last $N-1$ components of the vector $H_{N}^{\prime} \mathbf{a}^{T}$ at its first row. Both matrices $H_{N}^{\prime}$ and $H_{N}^{\prime \prime}$ may be obtained by Algorithm 1 with corresponding input vectors. Similarly more than two predefined vectors, orthogonal to each other, may be involved into the transform matrix.

\section{IMAGE COMPRESSION ALGORITHMS}

A traditional block transform-based image compression scheme (see Figure 4) applies a fixed transform to every subimage $(8 \times 8$ block $)$ and implements the actual compression in the transform domain by quantizing the transform coefficients followed by lossless encoding of the quantized values. Obtained bitstream is sent to the decoder, which implements inverse procedures to obtain an estimate of the encoded image. This scheme is motivated by the fact that, for most of natural images, content within small blocks is rather flat meaning high correlation between image pixels. When a proper transform (typically 2D separable DCT) is applied to such a flat block the largest portion of energy tends to be concentrated within relatively small number of transform coefficients. The better the transform concentrates the energy, the better is the performance of the compression scheme meaning closeness of the estimate on the decoder output to the original image on the encoder input at given compression ratio (ratio between the number of bits representing the original image and the number of bits representing the compressed image). The known fixed transforms, including DCT, perform rather well for flat image blocks but typically fail for active blocks with low correlation between pixels. The performance of the block-based image compression scheme could potentially be improved by possibility to use several transforms each specifically synthesized for a certain type of blocks. Parametric transforms of Section 2 and the method of synthesizing them may offer such an opportunity.

Based on (8), it is clear that parametric transforms are able to concentrate arbitrary image block energy into just one transform coefficient, which would be the ideal energy concentration. For this, however, a transform should be synthesized per each column and per each row of an image block used as generating vectors of these transforms. Applying these transforms to the image block so that each of them is applied to its generating vector would result into a matrix with only nonzero entry at the up-left corner. However, there is also maximally large overhead in such an approach since all the generating vectors, that is whole the original input image block, should also be sent to the decoder.

The overhead may and should be reduced by compromising the energy concentration property. There are different opportunities for such a tradeoff. In a priori research we have compared several approaches with extensive simulations. Currently the following approaches described in Algorithms 2 and 3 were considered being suitable ones. In both approaches, parametric transforms are synthesized according to generating vectors that are obtained as averages of differently formed image subvectors (block rows and columns). The basic (experimentally confirmed) assumption here is that average of several vectors ( say $x_{i}, i=1, \ldots, n$ ) having similar properties tends to preserve shapes of these vectors. Therefore, the parametric transform synthesized according to the generating vector being an average of several vectors, say $x_{i}, i=1, \ldots, n$, would still efficiently concentrate energy of each of the vectors $x_{i}, i=1, \ldots, n$. In order to classify image subvectors (block rows and columns) the efficiency of DCT-based compression could be used.

Below, two image compression schemes are proposed $[24,25]$. In both schemes, the fixed classical DCT is used in combination with different parametric Haar-like transforms, 


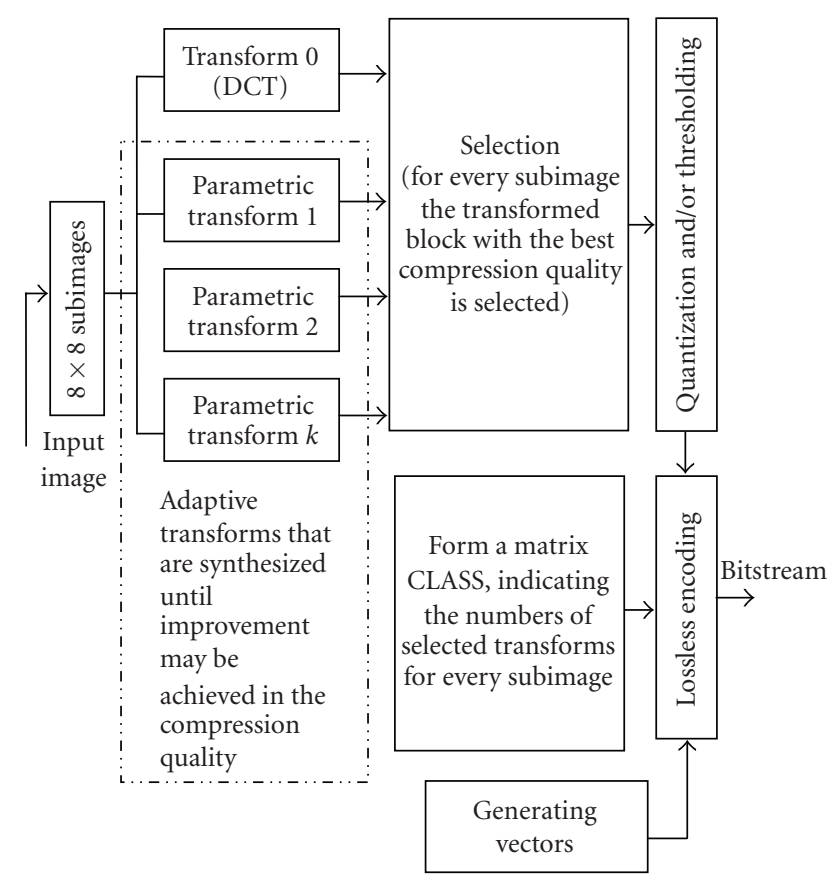

FIGURE 5: Image coder of proposed compression algorithm.

each synthesized and adapted to a certain type of blocks. The proposed compression schemes have performance that cannot be worse than a DCT-based scheme.

\subsection{Iterative image compression scheme (IICS)}

The first proposed scheme is iterative one where the classical DCT is used at the first iteration and iteratively synthesized Haar-like transforms are used at the following iterations. The main idea of the algorithm is to apply few parametric Haarlike transforms, each specifically designed for a specific class of blocks. Transforms are iteratively synthesized based on the blocks for which lowest compression efficiency was achieved with the previous transform (DCT being the initial one). The iterative process is continued as long as a worth-while overall compression efficiency improvement is observed for the whole image. The diagram of the image coder is depicted by Figure 5. A more detailed, step-by-step description of the proposed scheme follows.

Algorithm 2 (IICS).

Step 1. Apply the traditional image compression scheme as shown in Figure 4. In our current implementation, the input $N \times M$ image is partitioned into $8 \times 8$ blocks, which are DCT transformed, uniformly quantized, and hard thresholded. Then, zig-zag scanning followed by lossless encoding is applied to form the output bitstream.

Step 2. Initialize an $n \times m$ matrix CLASS $(n=N / 8, M=m / 8)$ with all the zero entries. The matrix CLASS will iteratively be updated to contain the indices of transforms assigned to the blocks. The DCT is indexed by zero and the transforms synthesized at the following iterations are indexed by the numbers of corresponding iterations.

Step 3. Measure the compression efficiency for every block and collect blocks with worst compression efficiency values. To measure the compression efficiency for a block $B$ we used the following functional:

$$
\mathrm{CE}(B)=\frac{1}{c_{1} \cdot \mathrm{SE}(B)+c_{2} \cdot \mathrm{NB}(B)},
$$

where $c 1, c 2$ are parameters that control the relative significance between the compression ratio and the distortion; NB is the number of bits representing the block after coding (DCT, quantization and lossless encoding); SE is the absolute difference between the original and the reconstructed image block. Higher CE value indicates better compression. The relation between parameters $c 1$ and $c 2$ is such that

$$
c_{1}=\frac{c}{\max _{\operatorname{all} B}\left(\mathrm{SE}_{\mathrm{DCT}}(B)\right)}, \quad c_{2}=\frac{1-c}{\max _{\operatorname{all} B}\left(\mathrm{NB}_{\mathrm{DCT}}(B)\right)},
$$

where $0<c<1, \operatorname{SE}_{\mathrm{DCT}}(B)$ and $\mathrm{NB}_{\mathrm{DCT}}(B)$ are corresponding characteristics obtained by DCT coding the block $B$. Note that larger value of $c$ means more significance of achieving less distortion rather than achieving higher compression.

To collect blocks with the worst coding efficiency we use the condition

$$
\mathrm{CE}(B)<\alpha \cdot \operatorname{mean}_{\text {all } B}\{\mathrm{CE}(B)\}
$$

where $\alpha$ is a parameter. Blocks satisfying this condition were collected.

Step 4. Synthesize a new 2D parametric transform adapted for the blocks collected at Step 3. In current implementation, a separable 2D transform is synthesized such that each block is multiplied by a matrix $H^{(1)}$ from the left-hand side, and by another matrix $H^{(2)}$ from the right-hand side. The parametric transform $H^{(1)}$ is generated by the transposed mean column and the transform $H^{(2)}$ is generated by the mean row of the collected worst blocks.

Step 5. Apply the new synthesized transform to every block of the image. For every block, if the current transform gives a smaller value of $\mathrm{CE}(B)$ than the previous transform, assign the current transform to the given block. Update the matrix CLASS correspondingly. Note that in calculation of $\mathrm{CE}(B)$ the overhead bits for presenting the generating vectors and the CLASS matrix must be taken into account.

Step 6. Lossless encode the generating vectors and the CLASS matrix. Compare the compression efficiency for the whole image (taking into account the overhead) to the compression efficiency obtained at previous iteration. If there is enough improvement, then go to Step 3. Otherwise, send the results of the previous iteration (the compressed blocks, lossless coded generating vectors-and lossless-coded CLASS matrix) to the storage or channel. 


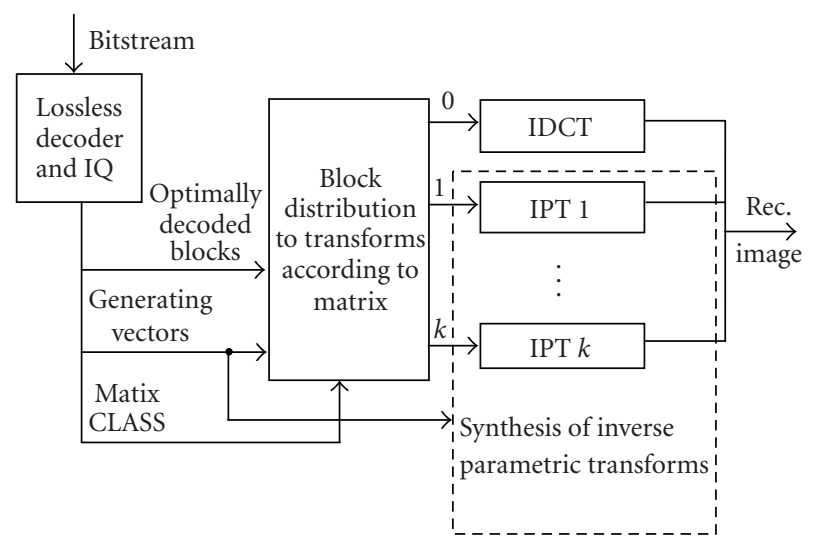

Figure 6: General scheme of an image decoder for two proposed compression algorithms.

Decoder performs the reconstruction of the blocks according to the matrix CLASS, elements of which show to decoder the number of the received generating vector based on which decoder constructs inverse transform and implements the reconstruction of the image. The decoder of the proposed scheme is illustrated in Figure 6.

\subsection{Multiple transform-based image compression (MTIC) algorithm}

The idea of the second proposed scheme is to apply multiple transforms to each image block and to select the best one according to a quality functional. Multiple transform-based image coder diagram is shown in Figure 7. The decoder in this algorithm performs the same procedure as in the previous scheme (see Figure 6). A more detailed, step-by-step description of the proposed scheme follows.

Algorithm 3 (MTIC).

Step 1. At this step a JPEG-like or DCT-based image compression scheme is applied to the given $N \times M$ image.

Step 2. For each nonoverlapping $8 \times 8$ image block $B$ of the input image the quality functional $\mathrm{CE}(B)$ according to $(21)$ is calculated. The values of the quality functional for all blocks are collected in a CEM matrix of order $(N / 8) \times(M / 8)$ which is then scaled to contain entries between zero and one. Thus, the matrix $Q M$ contains coding efficiency information obtained after the DCT-based compression of the original image.

Step 3. This is the block classification stage. At first, the range [min, max] is determined, where min and max indicate the minimal and maximal values of the $Q M$ matrix, respectively. Then, a series of subdivisions is applied to the range such that at each time the left half of previous division is selected for the following subdivision. Currently we apply only three subdivisions since the more the number of subranges, the more overhead and the complexity of the algorithm. Blocks corre- sponding to one subrange are collected together. Therefore, after classification we will have four types of blocks corresponding to four sequential subranges. Note that, on the average, smoother image blocks will fall into the subranges on the right side of the original range and less smooth blocks to the left subranges.

Step 4. Three new parametric 2D transforms are synthesized based on blocks collected to the lefter subranges. For every separable parametric $2 \mathrm{D}$ transform $H(i), i=1,2,3$, a matrix $H_{i}^{(1)}$, that is multiplied to a block from the left-hand side and another matrix $H_{i}^{(2)}$ that is multiplied from the right-hand side are synthesized. The parametric transform $H_{i}^{(1)}$ is generated by the transposed mean column and the transform $H_{i}^{(2)}$ is generated by the mean row of the collected blocks within $i$ th subrange. Therefore, three parametric $2 \mathrm{D}$ transforms are synthesized which correspond to three subranges.

Step 5. Initialize $n \times m$ matrix CLASS $(n=N / 8, m=M / 8)$ that will contain the indices of transforms assigned to the blocks. DCT is indexed by zero and the three newly synthesized transforms are indexed from 1 to 3 .

Step 6. Every $8 \times 8$ block is processed by four transforms, that is, 2D DCT and the three new synthesized parametric 2D transforms, are applied to every image block. Then, all transformed blocks are quantized, hard thresholded, and entropy encoded. Here the best transform out of four is selected for each block according to the quality functional CE (see (21)).

Step 7. Image blocks which are compressed with the "best" selected transforms are collected in a separate matrix. Since there is an overhead due to using parametric transforms, the question of "benefit" and "cost" of the parametric transforms becomes crucial. To solve this problem we need to verify the following condition: if the total "benefit" from using certain parametric transform over all blocks where it was selected is less than the cost of using that transform, it is not used. Every block where this transform was selected as the "best" is replaced with the DCT processed block. Otherwise, if the "benefit" is more than the cost, the corresponding transform is used.

Step 8. Update the block classification matrix CLASS correspondingly.

Step 9. The optimally coded blocks, the lossless-coded generating vectors, and the lossless-coded CLASS matrix represent the information, which is sent to the storage or channel.

Note that the DCT transform was indexed by 0 and the parametric transforms synthesized at Step 5 were indexed by the numbers from 1 to 3 . Therefore, the elements of CLASS matrix were $0,1,2,3$. One can see that the larger number of fragmentations was used in synthesizing Haar-like transforms, the larger number of bits is needed to represent the CLASS matrix resulting in a larger overhead. However, at Step 7 transforms whose benefit could not cover this overhead were removed and the CLASS matrix was accordingly 


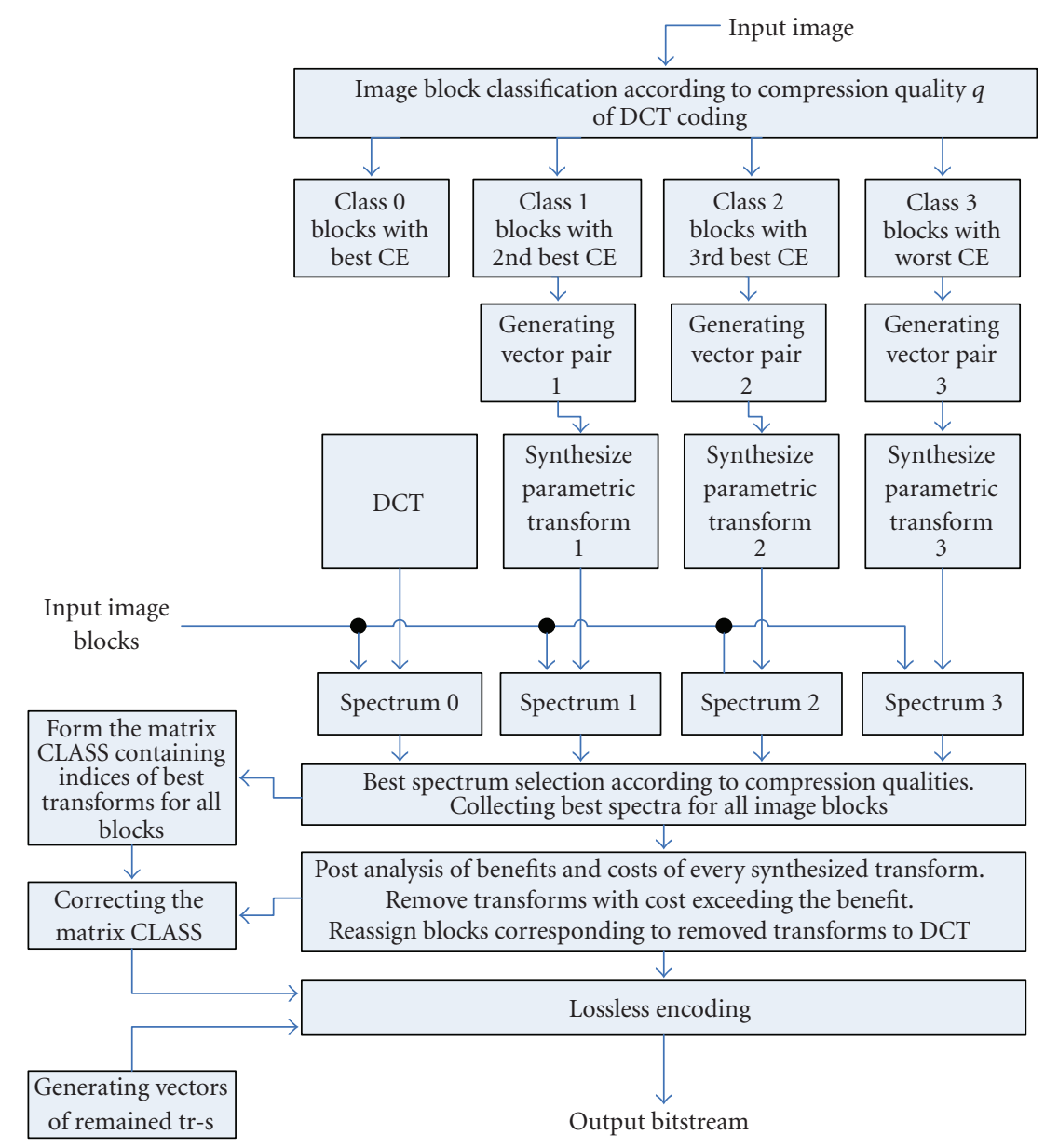

FIgURE 7: Multiple transform-based image coder.

updated at Step 8. Therefore, the overhead's influence to the overall coding quality can never be larger than the improvement due to using the final set of parametric transforms, that is, the performance of the proposed algorithm cannot be worse than that of the pure DCT-based algorithm.

\section{SIMULATIONS}

Both parametric transform-based image compression schemes were experimented on different test images and compared to the similar image compression scheme based on fixed DCT.

Some results obtained by the iterative compression scheme (Algorithm 2) are presented in Table 1. In this table, the results of the first iteration (presented in first rows for each image) correspond to the DCT-based compression scheme. Next iterations correspond to the case where parametric Haar-like transforms are also used. The last column in Table 1 presents parameters used in the experiments where $c$ is the constant used in compression efficiency functional calculation (see (21), (22)), $\alpha$ is the constant used to check "compressability" of the given block according to (23), and $Q$ is the quantization step that controls the bitrate in com-
TABLE 1: Simulation results of Algorithm 2 (IICS).

\begin{tabular}{l|c|c|c|c}
\hline Image & Iterat. & $\begin{array}{l}\text { Comp. } \\
\text { ratio }\end{array}$ & PSNR & Parameters \\
\hline \multirow{3}{*}{ Cameraman } & 1 & 6.29 & 38.17 & $c=0.05$ \\
& $\mathbf{2}$ & $\mathbf{6 . 3 0}$ & $\mathbf{3 8 . 5 2}$ & $\alpha=1$ \\
& 3 & 6.28 & 38.54 & $Q=12.8$ \\
\hline \multirow{3}{*}{ Medicalim } & 1 & 4.35 & 45.78 & $c=0.9$ \\
& $\mathbf{2}$ & $\mathbf{4 . 3 9}$ & $\mathbf{4 6 . 1 4}$ & $\alpha=0.8$ \\
& 3 & 4.35 & 46.19 & $Q=4$ \\
\hline \multirow{3}{*}{ Kidney } & 1 & 20.15 & 41.67 & $c=0.6$ \\
& 2 & $\mathbf{2 0 . 7 4}$ & $\mathbf{4 2 . 0 5}$ & $\alpha=1.2$ \\
Oesoph & 3 & 20.28 & 42.09 & $Q=12$ \\
\hline & $\mathbf{2}$ & 20.93 & 42.87 & $c=0.8$ \\
& 3 & 24.09 & $\mathbf{4 3 . 6 0}$ & $\alpha=0.9$ \\
& & & 43.60 & $Q=13.6$ \\
\hline
\end{tabular}

pressed images. Steps 1 to 6 of the proposed algorithm are iterated while the compression efficiency is increased by a predefined value of 0.2 at least. Iteration process is terminated when there is no enough increase in compression efficiency at 
TABLE 2: Simulation results of Algorithm 3 (MTIC).

\begin{tabular}{|c|c|c|c|c|}
\hline Image & Transform & $\begin{array}{l}\text { Comp. } \\
\text { ratio }\end{array}$ & PSNR & Parameters \\
\hline \multirow{2}{*}{ Compound } & DCT & \multirow{2}{*}{8.3} & 38.86 & \multirow{2}{*}{$\begin{array}{l}c=0.67, Q=20 \\
q 1=12 \\
q 2=10 \\
q 3=8\end{array}$} \\
\hline & Proposed & & 44.43 & \\
\hline \multirow{2}{*}{ Lena } & DCT & \multirow{2}{*}{8.7} & 34.73 & \multirow{2}{*}{$\begin{array}{l}c=0.58, Q=19.2 \\
q 1=24.96 \\
q 2=23.04 \\
q 3=7.68\end{array}$} \\
\hline & Proposed & & 35.58 & \\
\hline \multirow{2}{*}{ Cameraman } & DCT & \multirow{2}{*}{7.3} & 36.66 & \multirow{2}{*}{$\begin{array}{l}c=0.37, Q=16 \\
q 1=16 \\
q 2=14.4 \\
q 3=12.8\end{array}$} \\
\hline & Proposed & & 38.34 & \\
\hline \multirow{2}{*}{ Mandril } & DCT & \multirow{2}{*}{8.4} & 28.38 & \multirow{2}{*}{$\begin{array}{l}c=0.1, Q=36 \\
q 1=43.2 \\
q 2=39.6 \\
q 3=36\end{array}$} \\
\hline & Proposed & & 28.41 & \\
\hline
\end{tabular}

current iteration. Therefore, the final (the best) result of the proposed algorithm corresponds to the result of the penultimate iteration (highlighted with bold font type). One can see from Table 1 that, for approximately the same compression ratios, PSNR is increased up to penultimate iteration for all the images.

To analyze the performance of Algorithm 2, PSNR versus bitrate (bpp) plots were also obtained for several images. In these experiments, the values of parameters $c$ and $\alpha$ were fixed for every image and the parameter $Q$ was varied to achieve different bitrates. Figure 8 shows plots for several images. One can see that essential performance is achieved especially for the image "Compound."

Table 2 presents some results of experiments for Algorithm 3 in comparison with the DCT-based scheme. The last column indicates the parameter values used in the corresponding experiments. Here $c$ is the same as in Table 1 (see (21), (22)), $Q$ is the quantization step used in DCTbased coding (both at the first step of Algorithm 3 and in the reference DCT-based scheme), and $q 1, q 2, q 3$ are quantization steps used in association with corresponding three parametric transforms synthesized according to Algorithm 3. Table 2 illustrates the essential performance improvement for all the test images. Figure 9 shows PSNR versus bitrate plots of Algorithm 3 for different images.

To visualize the performance of the parametric-transform-based image compression schemes, Figure 10 illustrates comparative performance of Algorithms 2 and 3 with that of the DCT-based scheme for the medical image "kidney" (as known in [15], visual quality is the most important characteristic of medical image compression schemes).

It should also be noted that both Algorithms 2 and 3 do not only perform image compression but also implement block classification. Such classification is an important prop-

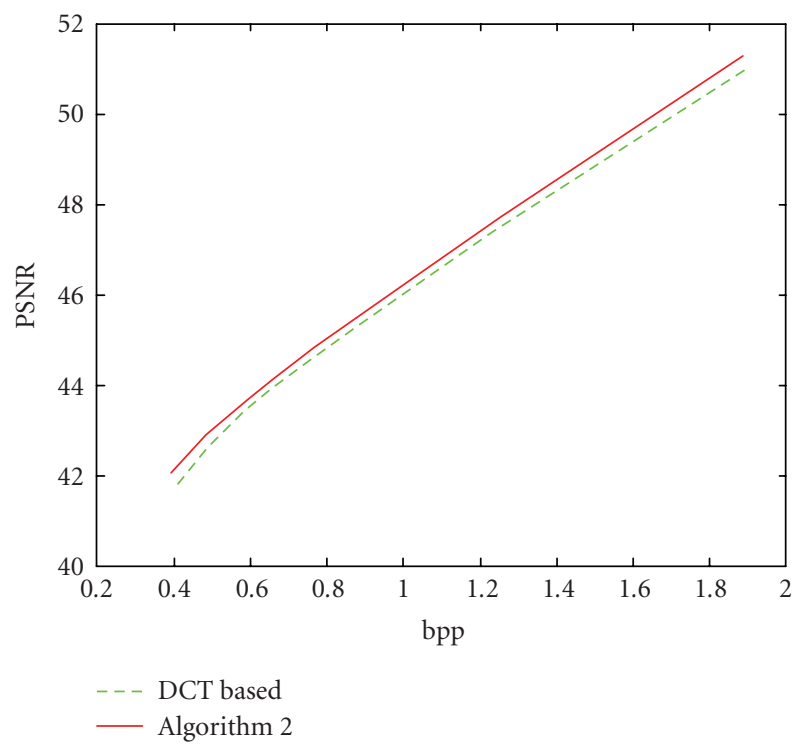

(a) Kidney image: Algorithm 2 versus DCT-based compression

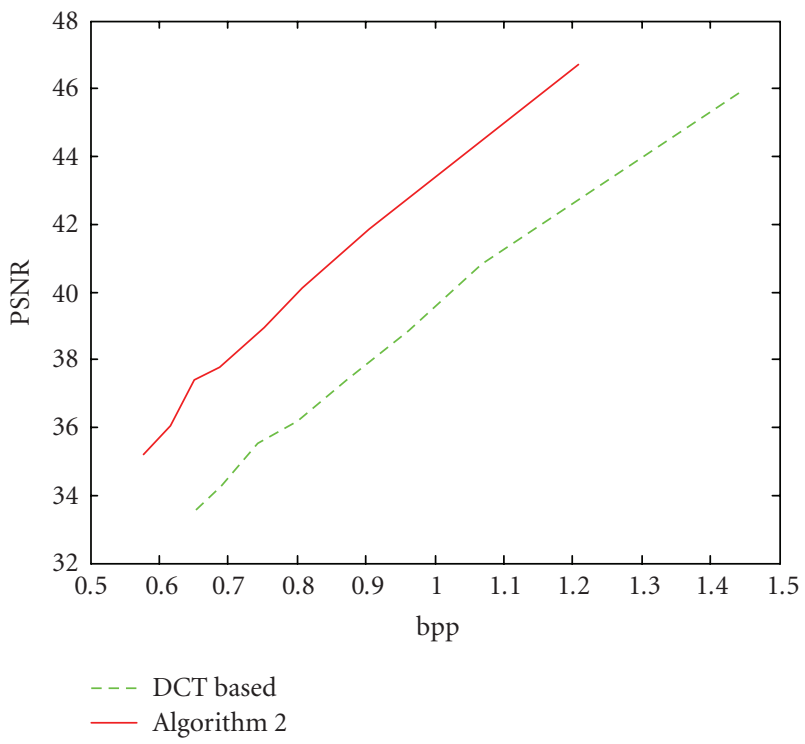

(b) Compound image: Algorithm 2 versus DCT-based compression

FiguRE 8: PSNR versus bitrate plots for images: (a) "Kidney" ( $c=$ $0.9, \alpha=1)$; (b) "Compound" ( $c=0.5, \alpha=1)$.

erty, especially for images composed of subimages of several types. Figure 11 presents plots of the matrices CLASS obtained by Algorithms 2 and 3 for the Compound image. The black pixels in this plot correspond to the use of DCT, while white pixels correspond to the use of parametric Haar-like transforms.

As can be seen from Figure 11, DCT is associated with flat regions of the image, while new transforms perform around nonflat regions, which is the expected result. 


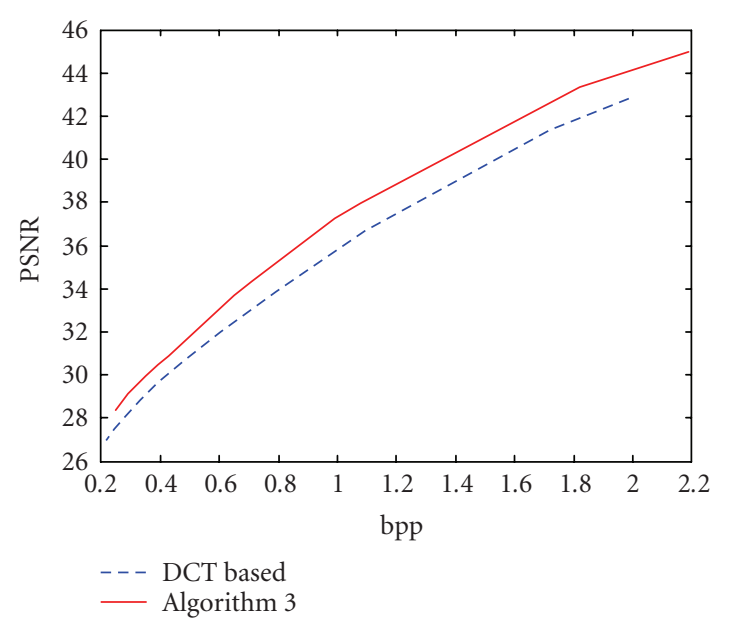

(a) Cameraman image: Algorithm 3 versus DCT-based compression

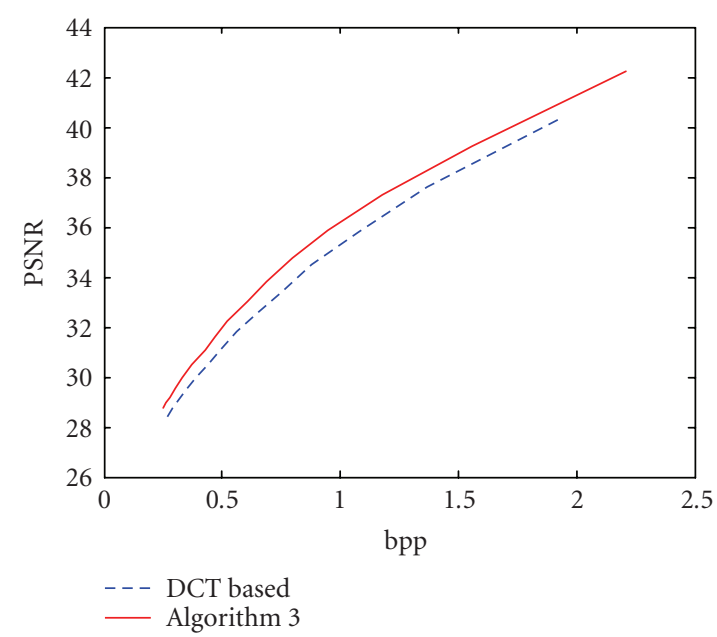

(b) Lena image: Algorithm 3 versus DCT-based compression

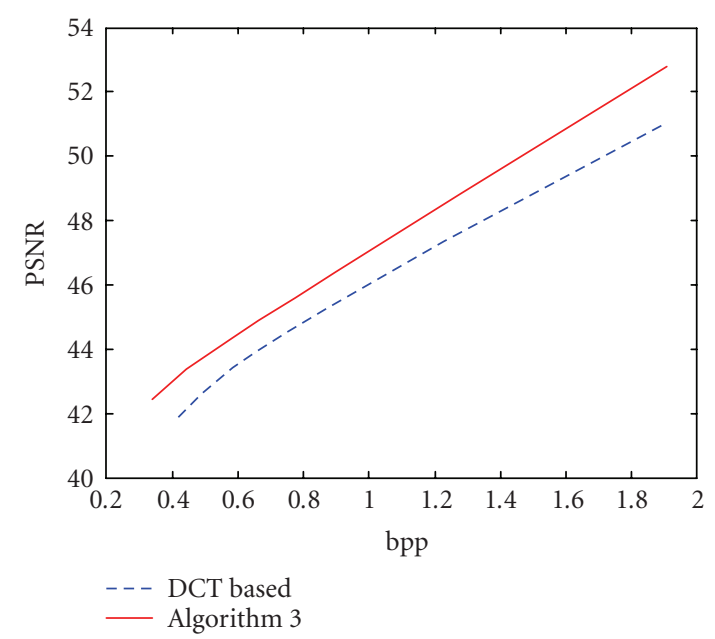

(c) Kidney image: Algorithm 3 versus DCT compression

FIGURE 9: PSNR versus bitrate plots for images: (a) "Cameraman;" (b) "Lena;" (c) "Compound." For all images $c=0.5, Q=8 \cdots 80$, $q 1=Q, q 2=0.9 Q, q 3=08 Q$.

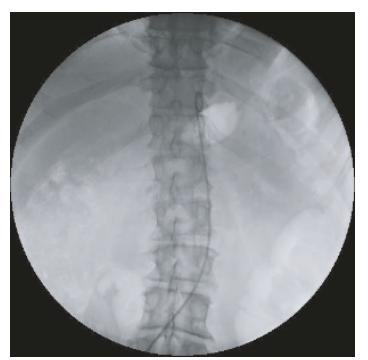

(a)

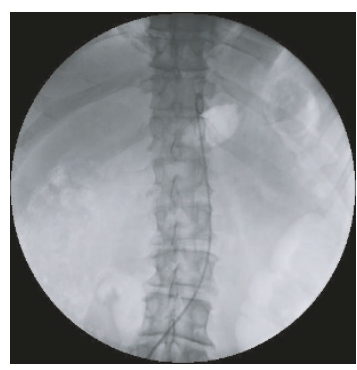

(c)

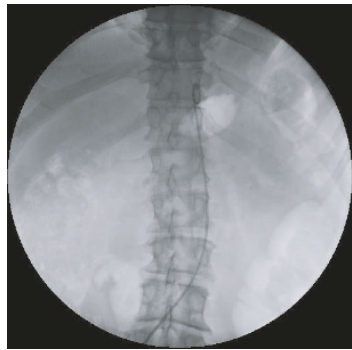

(b)

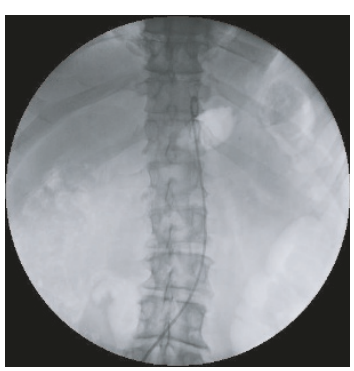

(d)
Figure 10: Medical image "Kidney": (a) the original image; (b) 6.53 times DCT compressed image; (c) 6.67 times compressed image with Algorithm 2; (d) 6.72 times compressed image with Algorithm 3.

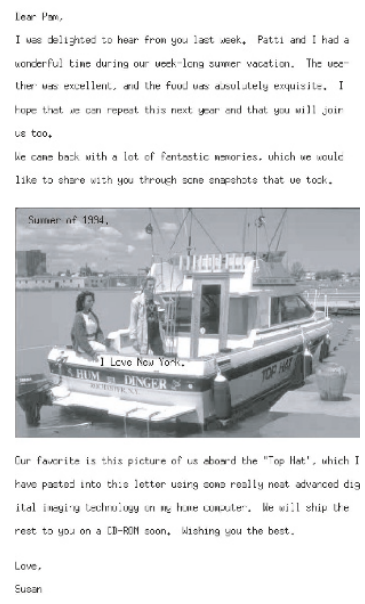

(a)
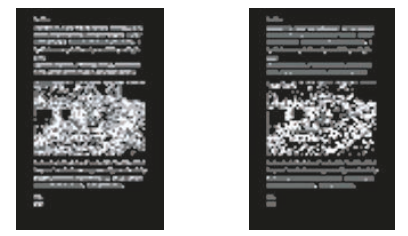

(b)

(c)
FIGURE 11: Results for "Compound" image: (a) original image; (b) matrix CLASS obtained by Algorithm 2; (c) matrix CLASS obtained by Algorithm 3.

\section{UNIFIED ARCHITECTURE FOR SYNTHESIS AND IMPLEMENTATION OF PARAMETRIC TRANSFORMS}

Many different architectures for fixed transforms and few architectures for families of transforms have been proposed in 


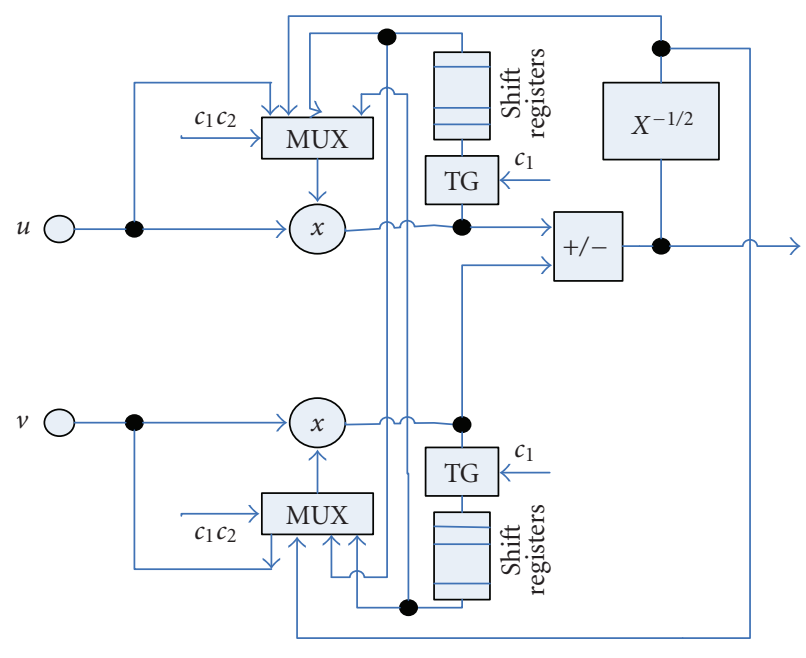

FIGURE 12: The structure of the generic PE for unified architectures for parametric transform synthesis and implementation.

the literature. However, to the best of our knowledge, no unified architecture that may synthesize a transform according to a set of parameters and then implement the synthesized transform is known. In this section, we propose architectures that operate in two modes: synthesis mode and implementation mode. In the synthesis mode, the architecture has a desired vector $\mathbf{h}$ in its input and computes the parameters (spectral kernels) that correspond to an orthogonal transform of a given type (Haar-like, Hadamard-like, etc.). These parameters are stored in registers and then are used in the implementation mode where the synthesized transform is applied to an arbitrary input vector.

Most of the architectures for fixed transforms or transform families are designed by mapping the flowgraph of the corresponding fast transform algorithm into architectures of one or another type (pipelined, iterative, parallel pipelined, folded, etc.). Vertical projections of the flowgraph to chains of processing elements (PEs) that implement the "butterflies" lead to pipelined designs. In pipelined designs, stages of the flowgraph are performed in parallel while the butterflies of a stage are performed sequentially. Typically, shift registers, delays, and multiplexers are used to implement permutations according to the sets of edges of the corresponding flowgraph. Horizontal projections to arrays of PEs lead to iterative processors. Permutations according to the sets of edges are implemented within the interconnection network between PEs. Actually, the flowgraph itself where the blocks corresponding to spectral kernels are replaced by PEs may, in principle, be considered as a parallel-pipelined (or stream) architecture. To reduce the size of the architectures, usually folding is applied where the number of PEs is used, so that each PE implements a number of "butterflies."

Here, we will not go into deeper architectural details but will concentrate on a generic PE structure that, when used in any architecture for a fast transform algorithm involving "butterflies," allows synthesizing new transforms based on
Algorithm 1 and at the same time efficiently implements the "butterflies." The generic PE structure is shown on Figure 12. It operates in two modes. In synthesis mode, the control signal $c_{1} c_{2}$ to multiplexers is first set to $c_{1} c_{2}=00$ so that its every input is multiplied to itself. The results of multiplications (squarings) are added within the adder and the sum is then inverted within the "Inv $(1 / \mathrm{x})$ " block. Then the control signal to the multiplexers is set to $c_{1} c_{2}=01$ so that the result $\left(1 /\left(u^{2}+v^{2}\right)\right)$ is sent to the two multipliers which obtain the two desired parameters $u /\left(u^{2}+v^{2}\right)$ and $v /\left(u^{2}+v^{2}\right)$ which are stored in the shift register file (at this point the transmitter gate "TG" is open). In the shift register file there are as many registers as many "butterflies" the PE will be implementing in the implementation mode. When parameters for all butterflies assigned to the given PE are computed, the control signal is set to $c_{1} c_{2}=10$ which switches PE to the implementation mode. The parameters from shift registers are sent to multipliers so that the first row of the spectral kernel is multiplied to the input vector. At the next step the control signal is set to $c_{1} c_{2}=11$ so that the second row of the spectral kernel is multiplied with the input pair. The results are sent to the next, within the given architecture, PE for computing the parameters of butterflies that correspond to that PE.

Note that the square root divider block within the PE is the most complicated block. Its complexity is approximately the same as of the divider (see, e.g., [26]). However, this block is out of the critical path in the implementation mode so that the speed of the proposed PE in this mode is approximately the same as if it was designed only to implement a fixed transform. It is much slower in the synthesis mode. In most of the applications synthesizing is applied much less frequently compared to the implementation of the transforms (see Algorithms 2 and 3). One way to exclude the square root divider block from the PE could be creating a look-up table (LUT) outside of the PE and reuse this LUT for every PE within the architecture for transforms. Efficiency of this approach depends on the working precision of the architecture and the size of the transforms being implemented. Another way to exclude this complex block could be based on computing the parameters of the transform outside of the hardware architecture for the transform and then "manually" tuning the PE to the desired transform by directly loading computed parameters to the shift registers. However, the alternative presented on Figure 12 allows also automatic generation of transform parameters meaning that the transform may automatically be adapted to the input signal or image.

\section{CONCLUSION}

A new class of parametric transforms was investigated in two new image compression schemes. Experimental results illustrated a moderate performance improvement for natural images and significant performance improvement for images of certain types such as medical images and complex images consisting of fragments of essentially different types. A methodology for developing VLSI architectures for synthesizing and implementation of the parametric transforms was also proposed. 


\section{REFERENCES}

[1] W. B. Pennebaker and J. L. Mitchell, JPEG Still Image Data Compression Standard, Van Nostrand Reinhold, New York, NY, USA, 1993.

[2] S. S. Agaian, "Optimal algorithms of fast orthogonal transforms and their implementation on computers," in Kibernetika I Vichislitelnaya Tekhnika, issue 2, pp. 231-319, Nauka, Moscow, Russia, 1986.

[3] S. S. Agaian, J. Astola, and K. Egiazarian, Binary Polynomial Transforms and Non-linear Digital Filters, Marcel Dekker, New York, NY, USA, 1995.

[4] S. S. Agaian and A. K. Matevosian, "Generalized Haar transforms and automation systems for testing quality of circuits," Acta Cybernetica, vol. 5, pp. 345-362, 1981.

[5] N. U. Ahmed and K. R. Rao, Orthogonal Transforms for Digital Signal Processing, Springer, Secaucus, NJ, USA, 1975.

[6] O. K. Ersoy, "A comparative review of real and complex Fourier-related transforms," Proceedings of the IEEE, vol. 82, no. 3, pp. 429-447, 1994.

[7] A. K. Jain, Fundamentals of Digital Image Processing, PrenticeHall, Englewood Cliffs, NJ, USA, 1989.

[8] A. D. Poularikas, Ed., The Transforms and Applications Handbook, CRC Press, Boca Raton, Fla, USA, 1996.

[9] H. S. Malvar, Signal Processing with Lapped Transforms, Artech House, Norwood, Mass, USA, 1992.

[10] M. V. Wickerhauser, Adapted Wavelet Analysis from Theory to Software, IEEE Press, A. K. Peters, Wellesley, Mass, USA, 1994.

[11] V. G. Labunets, "A unified approach to fast transformation algorithms," in Primeneniye Ortogonalnix Metodov pri Obrabotke Signalov i Analize System, pp. 4-14, UPI, Sverdlovsk, Russia, 1980.

[12] M. Traxtman and V. A. Traxtman, Osnovi Teorii Discretnix Signalov na Konechnix Intervalax, Sovetskoye Radio, Moscow, Russia, 1975.

[13] L. P. Yaroslavskiy, "Some questions of the theory of discrete orthogonal transforms of signals," in Cifrovaya Obrabotka Signalov I ee Primeneniya, pp. 33-71, Nauka, Moscow, Russia, 1981.

[14] C. Christopoulos, A. Skodras, and T. Ebrahimi, "The JPEG 2000 still image coding system: an overview," IEEE Transactions on Consumer Electronics, vol. 46, no. 4, pp. 1103-1127, 2000.

[15] D. H. Foos, E. Muka, R. M. Slone, et al., "JPEG 2000 compression of medical imagery," in Medical Imaging 2000: PACS Design and Evaluation: Engineering and Clinical Issues, vol. 3980 of Proceedings of SPIE, pp. 85-96, San Diego, Calif, USA, February 2000.

[16] N. Ponomarenko, V. Lukin, K. Egiazarian, and J. Astola, "DCT based high quality image compression," in Proceedings of the 14th Scandinavian Conference on Image Analysis (SCIA '05), pp. 1177-1185, Joensuu, Finland, June 2005.

[17] A. I. Solodovnikov, I. I. Kanatov, and A. M. Spivakovskii, "Synthesis of orthogonal bases from a generalized spectral kernel," in Voprosy Teorii Sistem Avtomaticheskogo Upravleniya, vol. 2, pp. 99-112, LGU, Leningrad, Russia, 1978.

[18] A. I. Solodovnikov, "Synthesis of complete orthonormal systems of functions having fast transform algorithm," in Voprosy Teorii System Avtomaticheskogo Upravleniya, vol. 4, pp. 94-105, LGU, Leningrad, Russia, 1978.

[19] H. C. Andrews and K. L. Caspary, "A generalized technique for spectral analysis," IEEE Transactions on Computers, vol. 19, no. 1, pp. 16-25, 1970.
[20] S. S. Agaian and D. Z. Gevorkian, "Complexity and parallel algorithms of discrete orthogonal transforms," in Kibernetika I Vichislitelnaya Tekhnika, issue 4, pp. 124-169, Nauka, Moscow, Russia, 1988.

[21] S. S. Agaian and D. Gevorkian, "Synthesis of a class of orthogonal transforms: parallel SIMD-algorithms and specialized processors," Pattern Recognition and Image Analysis, vol. 2, no. 4, pp. 394-408, 1992.

[22] S. Minasyan, D. Guevorkian, and H. Sarukhanyan, "On parameterized fast Haar- and Hadamard-like transforms of arbitrary order," in Proceedings of the 3rd International Conference on Computer Science and Information Technologies (CSIT '01), pp. 294-298, Yerevan, Armenia, September 2001.

[23] S. Minasyan, D. Guevorkian, S. S. Agaian, and H. Sarukhanyan, "On "slant-like" fast orthogonal transforms of arbitrary order," in Proceedings of the 4th EURASIP IEEE Region and International Symposium on Video/Image Processing and Multimedia Communications (VIPromCom '02), pp. 309-314, Zadar, Croatia, June 2002.

[24] S. Minasyan, J. Astola, and D. Guevorkian, "An image compression scheme based on parametric Haar-like transform," in Proceedings of IEEE International Symposium on Circuits and Systems (ISCAS '05), vol. 3, pp. 2088-2091, Kobe, Japan, May 2005.

[25] J. Astola, S. Minasyan, and D. Guevorkian, "Multiple transform based image compression technique," in Proceedings of the 5th IASTED International Conference on Visualization, Imaging, and Image Processing, Benidorm, Spain, September 2005.

[26] P. Soderquist and M. Leeser, "Division and square root: choosing the right implementation," IEEE Micro, vol. 17, no. 4, pp. 56-66, 1997.

Susanna Minasyan graduated from Yerevan State Engineering University of Armenia (SEUA) where in 1996 she got her Diploma with honors in the field of automated systems of information processing and management and in 1998 she received her M.S. degree in mathematics. From 1998 to 2001 she worked as a lecturer at the Department of Mathematics, SEUA. From 2001 to 2004, she was with Institute for Informatics and Automation Problems, National Academy of Sciences of Armenia. In 2004, she moved to Tampere University of Technology (TUT), Finland, first as a Visiting Researcher at Tampere International Center of Signal Processing and then as a Ph.D. student and researcher. Her research areas include adaptive transform-based methods in signal and image processing, and spectral techniques.

Jaakko Astola received his Ph.D. degree in mathematics from Turku University, Finland, in 1978. From 1976 to 1977 he was with the Research Institute for Mathematical Sciences of Kyoto University, Kyoto, Japan. Between 1979 and 1987 he was with the Department of Information Technology, Lappeenranta University of Technology, Lappeenranta, Finland. In 1984 he worked as a Visiting Scientist in Eindhoven

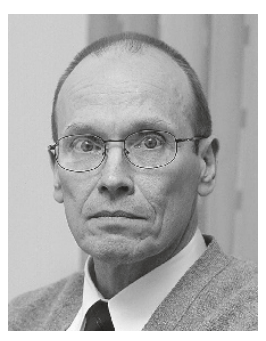
University of Technology, The Netherlands. From 1987 to 1992 he was Associate Professor in applied mathematics at Tampere University, Tampere, Finland. From 1993 he has been Professor of signal 
processing at Tampere University of Technology and is currently Head of Academy of Finland Centre of Excellence in Signal Processing leading a group of about 80 scientists. His research interests include signal processing, coding theory, spectral techniques, and statistics.

David Guevorkian received his M.S. degree (with honors) in applied mathematics from Yerevan State University, Armenia, in 1983, his Ph.D. degree in cybernetics and computational mathematics from Kiev State University, Ukraine, in 1987, and his Dr. Tech. degree in signal and image processing from Tampere University of Technology, Finland, in 1997. He was with the Institute for Problems of Informatics and

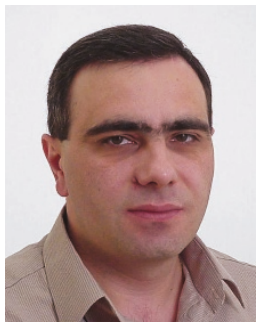
Automation (IPIA), National Academy of Sciences of Armenia, in 1983-1993. From 1993 to 2000 he worked at Signal Processing Laboratory, Tampere University of Technology, Finland. Since 2000, he has been with Nokia Research Center, where he is currently a Principal Scientist. He is an author or coauthor of more than 100 scientific publications and patents in the field of computational methods, signal and image processing, communications, and implementations. 